\title{
Restoring neuroprotection through a new preclinical paradigm: translational success for NA-1 in stroke therapy
}

\author{
Ryan INSTRUM ${ }^{1}$, Hong-shuo SUN ${ }^{1,2,3,4, *}$
}

Acta Pharmacologica Sinica (2013) 34: 3-5; doi: 10.1038/aps.2012.175; published online 24 Dec 2012

\begin{abstract}
$\mathrm{C}$ erebrovascular disease remains one of the leading causes of death and disability throughout the world, and with an aging population, prevalence rates are expected to rise dramatically over the coming years ${ }^{[1]}$. It is thus imperative that we develop effective treatment strategies to meet this challenge. Presently, the only established method of pharmacological stroke intervention is early reperfusion by way of thrombolytic agents, yet the narrow therapeutic window and high risk of complications associated with these drugs disqualify most patients from receiving them. This has led researchers to pursue neuroprotective strategies aimed at amplifying the capacity of neural tissue to tolerate ischemia, independent of blood flow restoration. However, all previous attempts to improve clinical outcomes through neuroprotection have been unsuccessful, and many have suggested abandoning the concept entirely ${ }^{[2]}$. After decades of failure, Michael Tymianski's group may have provided evidence and a translational paradigm capable of reestablishing neuroprotection as a viable avenue for stroke treatment $t^{[3]}$.
\end{abstract}

The role of glutamate in excitotoxic

Departments of ${ }^{1}$ Surgery, ${ }^{2}$ Physiology, and ${ }^{3}$ Pharmacology, ${ }^{4}$ Institute of Medical Science, Faculty of Medicine, University of Toronto, 1 King's College Circle, Toronto, Ontario, Canada M5S 1 A8

* Correspondence to: Prof Hong-shuo SUN

(hss.sun@utoronto.ca) cell damage following ischemia, particularly through its interaction with the $N$-methyl- $D$-aspartate receptor (NMDAR), has been well documented. For this reason, stroke research has focused mainly on achieving neuroprotection through the use of NMDAR antagonists. Many of these therapeutic agents generated encouraging experimental results in animal models, but each failed to demonstrate positive effects in humans during clinical trials ${ }^{[4]}$. Moreover, antagonists produced numer- ous psychological side-effects, and some even had deleterious consequences in terms of patient outcome. Glutamate is the primary neurotransmitter in the brain, thus appropriate NMDAR activity is required for normal physiological, behavioral and cognitive functioning, as well as for promoting pro-survival pathways. The reliance of the brain on NMDA signaling accounts for the myriad of problems associated with completely blocking the channels and its overall ineffectiveness as a treatment for
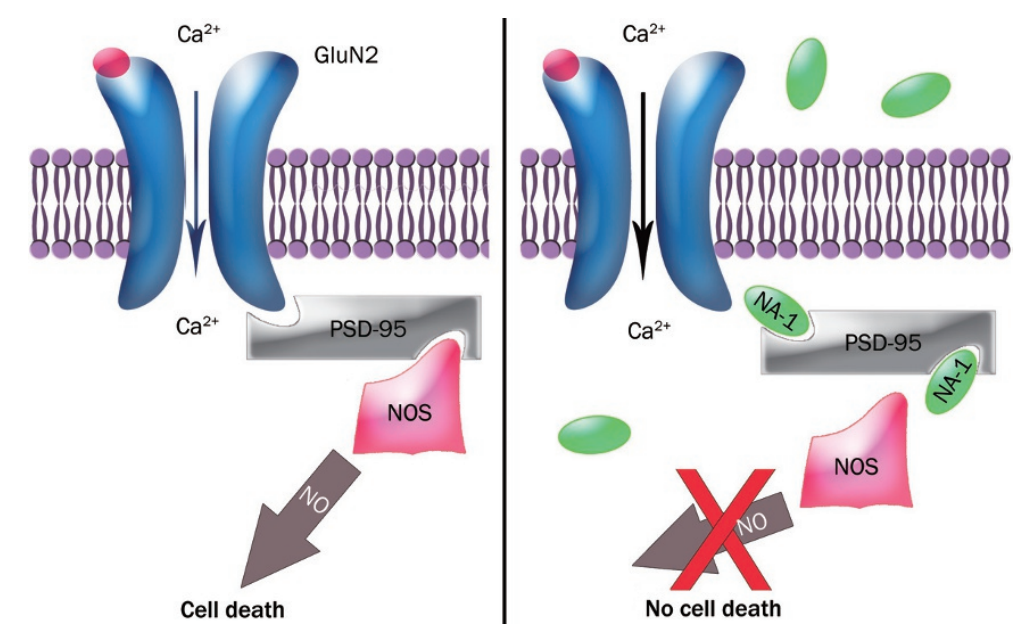

Figure 1. NA-1 targets postsynaptic density-95 (PSD-95) protein to inhibit NMDA-mediated excitotoxic signaling following ischemia. Left: PSD-95 is a scaffolding protein that links NMDA receptors (blue) to effector proteins such as nitric oxide synthase (NOS). Excess glutamate (red sphere) raises intracellular calcium levels leading to nitric oxide (NO) production and subsequent cell death. Right: NA-1 competitively inhibits PSD-95 by binding to its PDZ domains. This serves to disconnect NMDA currents from neurotoxic second messenger cascades, whereby preventing cell death while maintaining essential NMDA function. 
stroke.

Further use of NMDARs as a target for pharmacological action necessitated a higher level of specificity so as not to interfere with its essential functioning. This was achieved by way of postsynaptic density-95 (PSD-95) protein, a scaffolding protein that provides a link between NMDARs and effectors such as NOS that mediate neurotoxic signaling. Suppressed genetic expression and competitive inhibition of PSD-95 in vitro were both shown to protect neurons from NMDAR-mediated excitotoxicity and to suppress NO production, while normal NMDAR functioning was maintained $^{[5]}$. Targeting elements of the pathophysiological pathway further downstream provides a means of circumventing the negative consequences related to blocking receptors themselves.

By fusing a protein consisting of the final 9 C-terminal residues of the NMDA NR2B subunit to the cell-membrane transduction region of the HIV-1 Tat protein, a cell-permeable PSD-95 inhibitor (Tat-NR2B9c) was created ${ }^{[6]}$. Several studies demonstrated in vivo that TatNR2B9c indeed had neuroprotective effects (Figure 1) when administered following middle cerebral artery occlusion (MCAO), including a permanent $\mathrm{MCAO}$ model with severe hyperthermia ${ }^{[6,7]}$. These findings showed that compared to saline controls, infarct volume was reduced in Tat-NR2B9c-treated groups, and that these animals also had better functional recovery. Further trials using diffusion-weighted imaging/ perfusion-weighted imaging mismatch revealed that Tat-NRB29c attenuated lesion enlargement through the preservation of peri-infarct tissue, known as the ischemic penumbra, and did so without altering cerebral blood flow ${ }^{[8]}$. These results demonstrate that, in addition to its neuroprotective potential, TatNR2B9c could potentially be used in tandem with thrombolytic drugs to extend the therapeutic window, in which they are useful.

All previous neuroprotectant trials have failed to correlate laboratory findings into positive clinical outcomes, and this translational disconnect can, at least in part, be attributed to flawed preclinical experimental design. Critical assessments of failed studies have cited insufficient scope, a lack of clinically relevant methodology and failure to reproduce results in higher-order primate brains as reasons why human trials have been unsuccessful ${ }^{[2]}$. Subsequent trials involving Tat-NR2B9c were carefully planned, taking into consideration past shortcomings and the experimental framework established by the Stroke Academic Industry Roundtable (STAIR) committee $^{[3]}$.

To bridge the translational gap between rat models and human testing, Cook et $a l^{[9]}$ used higher-order nonhuman primates that are behaviorally and genetically similar to humans, and whom possess anatomically comparable gyrencephalic brains. A longitudinal 30-d study using MCAO was designed to assess infarct volume as well as neurobehavioral outcomes, sensorimotor functioning and the transcriptional capacity of penumbra tissue. Normalized data from Tat-NR2B9c-treated animals revealed a significantly reduced infarct volume relative to placebo [by $55 \%$ at $24 \mathrm{~h}$ (DWI) and by $70 \%$ at $30 \mathrm{~d}$ (T2-MRI) $]^{[9]}$. Similarly, animals treated with Tat-NR2B9c performed better on neurobehavioral and sensorimotor tests, and microarray analysis indicated that a lower proportion of genes in ischemic tissue were downregulated. Furthermore, in an attempt to simulate the use of Tat-NR2B9c as a means of extending therapeutic windows for thrombolytic drugs, additional trials were conducted with treatment 3-h after MCAO onset, and comparable results were obtained.

In the most recent set of experiments, Hill et al performed a phase-2, randomized, placebo-controlled, doubleblind study testing the pharmacological neuroprotective capacity of NA-1 (TatNR2B9c) in human patients undergoing endovascular aneurysm repair ${ }^{[3]}$. These Evaluating Neuroprotection in Aneurysm Coiling Therapy (ENACT) trials were conducted at 14 hospitals throughout the US and Canada. Peri-procedural iatrogenic stroke occurs in the majority of these surgical interventions creating multiple regions of infarct throughout the brain. This offered researchers the opportunity to test the viability of NA-1 as a neuroprotectant by administering the drug intravenously directly following surgery. The results of the study indicate that, compared to placebo, patients receiving NA-1 treatment sustained fewer lesions (DWI and FLAIR MRI) ${ }^{[3]}$. Secondary analysis of the data showed that patients with ruptured aneurysms had reductions in infarct volume and scored better on the National Institute of Health stroke scale than did those receiving saline-control ${ }^{[3]}$. These data closely resemble those obtained from a concurrent ENACT study in gyrencephalic non-human primates, and speak to the translational predictive value of their preclinical trials ${ }^{[10]}$.

Tymianski's group has provided indisputable evidence that neuroprotection is attainable in humans. This lends credibility to a principle that has been under siege due to decades of experimental disappointment. Larger-scale clinical trials will still be necessary to provide the statistical power needed to corroborate the entire story. The nature of the study does not provide the heterogeneity or practical time-frame for NA-1 administration, which are required to generalize these findings to all instances of acute ischemic stroke. However, many endovascular procedures pose the risk of iatrogenic stroke injury, and attenuating such stroke sequelae would be invaluable in its own right. Furthermore, the most powerful aspect of the studies is the unprecedented scope and rigor of its experimental design. The preclinical paradigm utilized in these trials may be capable of bridging the translational gap in neuroprotection research to allow for future clinical success in stroke treatment.

1 Roger VL, Go AS, Lloyd-Jones DM, Benjamin EJ, Berry JD, Borden WB, et al. Heart disease and stroke statistics -2012 update : a report from the American Heart Association. Circulation 2012; 125: e2-220.

2 O'Collins VE, Macleod MR, Donnan GA, Horky LL, van der Worp BH, Howells DW. 1,026 Experimental treatments in acute stroke. Ann Neurol 2006; 59: 467-77. 
3 Hill MD, Martin RH, Mikulis D, Wong JH, Silver FL, Terbrugge KG, et al. Safety and efficacy of NA-1 in patients with iatrogenic stroke after endovascular aneurysm repair (ENACT): a phase 2, randomised, double-blind, placebo-controlled trial. Lancet Neurol 2012; 11: 942-50.

4 Ikonomidou C, Turski L. Why did NMDA receptor antagonists fail clinical trials for stroke and traumatic brain injury? Lancet Neurol 2002; 1 : 383-6.

5 Sattler R, Xiong Z, Lu WY, Hafner M, MacDonald JF, Tymianski M. Specific coupling of NMDA receptor activation to nitric oxide neurotoxicity by PSD-95 protein. Science 1999; 284: 1845-8.

6 Aarts M, Liu Y, Liu L, Besshoh S, Arundine M, Gurd $J W$, et al. Treatment of ischemic brain damage by perturbing NMDA receptor-PSD-95 protein interactions. Science 2002; 298: 846-50.

7 Sun HS, Doucette TA, Liu Y, Fang Y, Teves L, Aarts $\mathrm{M}$, et al. Effectiveness of PSD95 inhibitors in permanent and transient focal ischemia in the rat. Stroke 2008; 39: 2544-53.

8 Bratane BT, Cui H, Cook DJ, Bouley J, Tymianski M, Fisher M. Neuroprotection by freezing ischemic penumbra evolution without cerebral blood flow augmentation with a postsynaptic density-95 protein inhibitor. Stroke 2011; 42: 3265-70.

9 Cook DJ, Teves L, Tymianski M. Treatment of stroke with a PSD-95 inhibitor in the gyrencephalic primate brain. Nature 2012; 483: 213-8.

10 Cook DJ, Teves L, Tymianski M. A translational paradigm for the preclinical evaluation of the stroke neuroprotectant Tat-NR2B9c in gyrencephalic nonhuman primates. Sci Transl Med 2012; 4: $154 \mathrm{ral} 33$. 